\title{
ADSORPTION OF OXYGEN ON HF-ETCHED Si(111) SURFACES: XPS STUDY
}

\author{
R.J. IWANOWSKI \\ Institute of Physics, Polish Academy of Sciences \\ Al. Lotników 32/46, 02-668 Warsaw, Poland \\ AND J.W. SoBCZAK \\ Institute of Physical Chemistry, Polish Academy of Sciences \\ Kasprzaka 44/52, 01-224 Warsaw, Poland
}

\begin{abstract}
X-ray photoelectron spectroscopy studies of the $\mathrm{Si}(111)$ surfaces, after dilute HF etching and methanol rinse, are reported. These included a detailed analysis of the main core-levels ( $\mathrm{Si} 2 p, \mathrm{O} 1 s$ ) and the valence band spectra. The observed asymmetry of the $\mathrm{O} 1 \mathrm{~s}$ lines was attributed to two contributing subpeaks: the main (1) and the minor one (2), shifted $\approx 1.5 \mathrm{eV}$ to higher binding energies. Their relative intensity was found to depend on the air exposure time and on the take-off angle. The peaks were assigned to two different positions of surface oxygen: (1) O chemisorbed with methoxy group, (2) bridging $O$ atom. The valence band X-ray photoelectron spectroscopy spectra reveal the influence of surface states induced by the "chemisorbed O"-Si bond.
\end{abstract}

PACS numbers: 79.60.Dp

\section{Introduction}

A silicon crystal held in air has a surface oxide layer of the so-called native oxide, which must be usually removed prior to inserting the sample into processing equipment or an experimental setup chamber. Such deoxidation occurs in a final stage of wet chemical treatment by dipping Si crystal in dilute HF, followed by adequate rinse. X-ray photoelectron spectroscopy (XPS) has been commonly used for investigating the microscopic structure of the HF-etched silicon surface, including single crystalline [1-5] and porous $\mathrm{Si}$ (e.g. [6]).

In the present paper, XPS measurements (including angle-resolved (AR) mode) of the HF-etched and air-exposed $\mathrm{Si}(111)$ surfaces are reported in an effort to understand better the binding structure of oxygen on silicon surface at the early oxidation stage.

\section{Experiment}

The samples used in the experiment, cut from the $n$-type float zone (FZ) Si single crystal (grown at the Institute of Electronic Materials Technology, Warsaw), had rectangular parallelepiped shape of $20 \times 10 \times 0.5 \mathrm{~mm}^{3}$. Their front (111) surfaces were prepared by mechanical and chemical fine (device grade) polishing. 
The room temperature XPS measurements were performed in a Vacuum Generators Escalab 210 spectrometer with a $\mathrm{Mg} K_{\alpha}(1253.6 \mathrm{eV})$ irradiation source. In the standard configuration a detector axis was perpendicular to the sample surface (take-off angle, $\theta=90^{\circ}$ ). Enhanced surface sensitivity was reached for $\theta=20^{\circ}$. During the experiment the pressure in the spectrometer chamber was approximately $3 \times 10^{-10} \mathrm{Tr}$.

Before each XPS measurement, the samples were subjected to the final wet chemical etching in aqueous HF (10\%) solution, followed by the methanol bath. Typically, an air exposure of the treated specimens, prior to insertion into the spectrometer chamber, lasted about $4 \mathrm{~min}$.

\section{Results and discussion}

The results of the experiment are listed in Table. This includes a detailed analysis of the main core-level (i.e. Si $2 p$ and $\mathrm{O} 1 s$ ) spectra recorded for a typical sample after standard $(4 \mathrm{~min}$ ) air exposure (experiment $A$ ) and its twin sample $(B)$, cut from the adjacent part of the same Si wafer. In the latter case (experiment $B)$ the sample was subjected to longer $(15 \mathrm{~min})$ air exposure than the standard one. $B^{\prime}$ refers to the XPS spectra of the $B$ sample, detected for $\theta=20^{\circ}$.

\section{TABLE}

Summary of the core-level XPS analysis with simulation of the Si $2 p$ and O $1 s$ photoelectron lines and relative atomic ratio, $[0] /[\mathrm{Si}] . \Gamma$ denotes full width at half maximum.

\begin{tabular}{c|c|c|c|c|c|c|c}
\hline \hline Experiment & \multicolumn{3}{|c|}{$\mathrm{Si}(2 p)$} & \multicolumn{3}{c|}{$\mathrm{O}(1 s)$} & Atomic ratio \\
& $E_{\mathrm{B}}[\mathrm{eV}]$ & $\Gamma[\mathrm{eV}]$ & $\%$ & $E_{\mathrm{B}}[\mathrm{eV}]$ & $\Gamma[\mathrm{eV}]$ & $\%$ & $0.1 \mathrm{Si}]$ \\
\hline$A$ & 99.3 & 0.84 & 66.0 & 531.9 & 1.92 & 85.0 & 0.105 \\
& 99.9 & 0.84 & 34.0 & 533.4 & 2.27 & 15.0 & \\
$B$ & 99.0 & 0.94 & 66.0 & 532.1 & 1.93 & 71.0 & 0.830 \\
& 99.6 & 0.93 & 34.0 & 533.7 & 2.51 & 29.0 & \\
$B^{\prime}$ & 99.0 & 0.92 & 65.0 & 532.2 & 2.08 & 87.0 & 0.465 \\
$\left(\theta=20^{\circ}\right)$ & 99.6 & 0.91 & 35.0 & 533.8 & 2.03 & 13.0 &
\end{tabular}

It was generally found in the standard case $(A)$ that the Si $2 p$ core-level spectra do not exhibit (see [5]) any additional features indicative of a chemical shift toward higher binding energies $\left(E_{\mathrm{B}}\right)$, that is characteristic of Si surface covered (even partially) with suboxides and/or $\mathrm{SiO}_{2}$ (e.g. $[3,7,8]$ ) or with $\mathrm{SiF}_{x}$ [9]. Figure 1a illustrates the effect of longer air exposure (case $B, B^{\prime}$ ) on the Si $2 p$ spectrum recorded by the AR XPS. It is evident that, despite prolonged exposure, one observes here only residual contribution of different suboxides in the range 100-104 eV.

The numerical analysis of the Si $2 p$ and O $1 s$ spectra, performed by fitting them with Gaussian-Lorentzian component peaks (Table), provided more information about the structure of the partially oxidized Si surfaces studied. It can be 

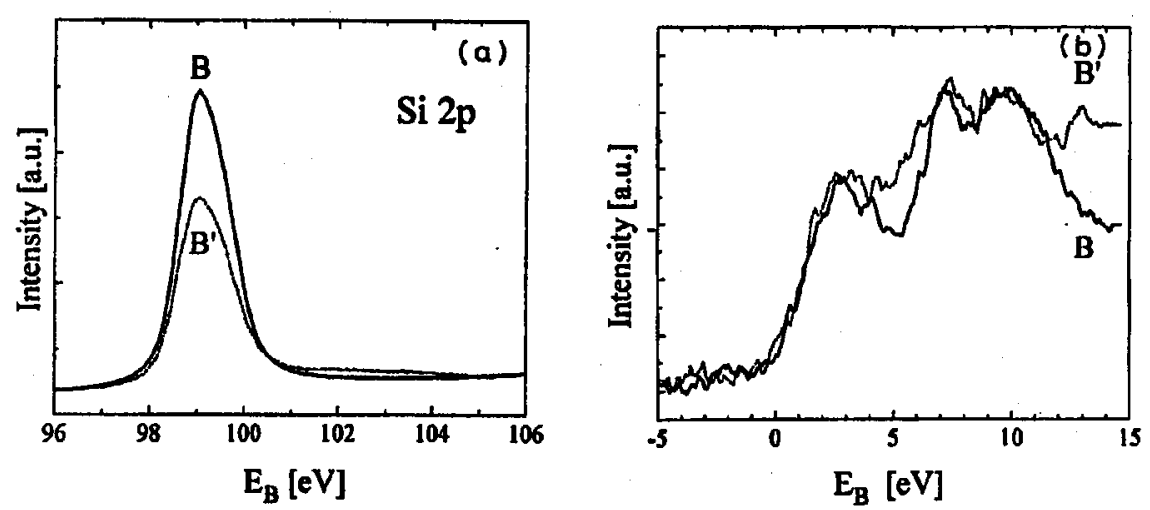

Fig. 1. Si $2 p$ XPS spectra (a) and the valence band XPS spectra (b) recorded at the take-off angle $90^{\circ}$ (solid line) and $20^{\circ}$ (dashed line) for the $\mathrm{Si}(111)$ surface, subjected to prolonged air exposure (case $B, B^{\prime}$ - respectively).

seen that the simulation of the recorded Si $2 p$ peaks (case $A$ and $B$ ) revealed a typical doublet split by $0.6 \mathrm{eV}$, with the intensity ratio $I\left(p_{3 / 2}\right) / I\left(p_{1 / 2}\right) \approx 2$ (e.g. [8]). The $\mathrm{O} 1 s$ core-level spectra recorded here exhibited slight asymmetry indicative of the initial oxidation stage of the Si surface (see e.g. [10]). Their simulations enabled us to distinguish (similarly as in [11]) two component peaks: the main peak at $E_{\mathrm{B} 1}=532 \mathrm{eV}$, and a minor one (at $E_{\mathrm{B} 2}$ ) shifted by about $1.5 \mathrm{eV}$ to higher binding energies.

Since the magnitude of $E_{\mathrm{B}}\left(\mathrm{O}_{1 s}\right)$ for the oxygen adsorbed on Si surface depends on the oxide thickness $[7,12]$, the observed binding energy of the main $\mathrm{O} 1 s$ component indicates that in our experiments $(A, B)$ the effective thickness of suboxides remains below $5 \AA$. However, a weak shift $(0.2 \mathrm{eV})$ of $E_{\mathrm{B} 1}$ and $E_{\mathrm{B} 2}$ to higher binding energies, resulting from increased air exposure (case $B$ ), is noticeable from Table. This correlates with an increased [O]/[Si] atomic ratio (Table). One should pay here special attention to the contribution of the $E_{\mathrm{B} 2}$ component to the total $\mathrm{O} 1 \mathrm{~s}$ line intensity $\left(I_{\mathrm{t}}\right)$. After prolonged air exposure $(B)$ the ratio $I\left(E_{\mathrm{B} 2}\right) / I_{\mathrm{t}}$ became nearly double. On the other hand, the relative contribution of peak 2 , measured in an increased surface sensitivity mode $\left(B^{\prime}\right)$, was significantly reduced.

The observed relative changes of both component peaks to $I_{\mathrm{t}}(\mathrm{O} 1 \mathrm{~s})$ could be explained by their assignment to two different nonequivalent binding positions of oxygen atom on the Si surface. The second peak $\left(E_{\mathrm{B} 2}\right)$ can be identified with bridging $\mathrm{O}$ atoms in the top $\mathrm{Si}$ layers whereas the main one $\left(E_{\mathrm{B} 1}\right)$ can be related to the chemisorbed $O$ atoms. Since the etching process applied here was followed by methanol bath it seems likely that oxygen remains chemisorbed in a methoxy group thus leading to formation of a stable surface species $\mathrm{SiOCH}_{3}[13,14]$. Our present assignment seems to contradict the one previously introduced in paper [11] - however, it should be emphasized here that its authors have not applied the AR-mode in their photoemission experiment. 
Additionally, Fig. 1b presents the valence band (VB) XPS spectra recorded at the take-off angle $90^{\circ}$ and $20^{\circ}$ for the Si surface, subjected to prolonged air exposure (case $B, B^{\prime}$ - respectively). The VB spectrum $B$ consists of three peaks, located near 3, 7.5 and $10 \mathrm{eV}$, which remain characteristic of $\mathrm{Si}(111)$ [6]. Its comparison with analogous spectrum measured for enhanced surface sensitivity $\left(B^{\prime}\right)$ clearly reveals the surface-related features located near $5 \mathrm{eV}$ and $13 \mathrm{eV}$. They can be attributed to the contribution of surface states induced by the "chemisorbed oxygen"-Si bond, denoted in literature by $\mathrm{O}_{b}$ and $\mathrm{O}_{\sigma}$ [15], respectively, thus remaining in agreement with the model considered.

\section{Conclusions}

The above analysis of the XPS data for the HF-etched Si(111) surfaces indicates (within our air-exposure time) that oxygen is adsorbed on Si surface with only residual coverage by $\mathrm{Si}$ oxides. It also enabled us to explain the asymmetry of the O $1 s$ XPS peak by a model of two contributing subpeaks and to achieve a suitable assignment to different bond configurations of oxygen with host atoms on Si surface.

\section{References}

[1] B.R. Weinberger, G.G. Peterson, T.C. Eschrich, H.A. Krasinski, J. Appl. Phys. 60, 3232 (1986).

[2] T. Takahagi, I. Nagai, A. Ishitani, H. Kuroda, Y. Nagasawa, J. Appl. Phys. 64, 3516 (1988).

[3] J.M.C. Thornton, R.H. Williams, Phys. Scr. 41, 1047 (1990).

[4] E.T.P. Benny, J. Majdi, J. Electron Spectrosc. Relat. Phenom. 58, 261 (1992).

[5] R.J. Iwanowski, J.W. Sobczak, B.J. Kowalski, Acta Phys. Pol. A 86, 825 (1994).

[6] R.P. Vasquez, R.W. Fathauer, T. George, A. Ksendzov, T.L. Lin, Appl. Phys. Lett. 60, 1004 (1992).

[7] J. Finster, D. Schultze, F. Bechstedt, A. Meisel, Surf. Sci. 152/153, 1063 (1985).

[8] F.J. Himpsel, F.R. McFeely, A. Taleb-Ibrahimi, J.A. Yarmoff, G. Hollinger, Phys. Rev. B 38, 6084 (1988).

[9] F.J. Himpsel, J.F. Morar, J.A. Yarmoff, J. Electrochem. Soc. 135, 2844 (1988).

[10] G. ivfargaritondo, J.E. Rowe, J.A. Jarmoff, Nuovo Cimento B 39, 781 (1977).

[11] G. Hollinger, J.F. Morar, F.J. Himpsel, G. Hughes, J.L. Jordan, Surf. Sci. 168, 609 (1986).

[12] M. Takakura, T. Ogura, T. Hayashi, M. Hirose, Jpn. J. Appl. Phys. 27, L2213 (1988).

[13] K. Edamoto, Y. Kubota, M. Onchi, M. Nishijima, Surf. Sci. 146, L533 (1984).

[14] M.N. Piancastelli, R. Zanoni, M. Marsi, G. Margaritondo, Solid Stade Commun. 79, 13 (1991).

[15] S. Ciraci, S. Ellialtioglu, S. Erkoc, Phys. Rev. B 26, 5716 (1982). 